\title{
Syndromic Multiplex Polymerase Chain Reaction (mPCR) Testing and Antimicrobial Stewardship: Current Practice and Future Directions
}

\author{
Theodore S. Rader IV ${ }^{1}$ (D) Michael P. Stevens ${ }^{2} \cdot$ Gonzalo Bearman $^{2}$ \\ Accepted: 27 January 2021 / Published online: 26 February 2021 \\ (C) The Author(s), under exclusive licence to Springer Science+Business Media, LLC part of Springer Nature 2021
}

\begin{abstract}
Purpose of Review Syndromic multiplex polymerase chain reaction (mPCR) panels offer the antimicrobial steward a rapid tool for optimizing and de-escalating antimicrobials. In this review, we analyze the role of syndromic mPCR in respiratory, gastrointestinal, and central nervous system infections within the context of antimicrobial stewardship efforts.

Recent Findings For all mPCR syndromic panels, multiple studies analyzed the pre-and-post implementation impact of mPCR on antimicrobial utilization. Prospective studies and trials of respiratory mPCR stewardship interventions, including diagnostic algorithms, educational efforts, co-testing with procalcitonin, and targeted provider feedback currently exist. For gastrointestinal and cerebrospinal fluid mPCR, fewer peer-reviewed reports exist for the use of mPCR in antimicrobial stewardship. These studies demonstrated an inconsistent trend towards decreasing antibiotic use with mPCR. This is further limited by a lack of statistical significance, the absence of controlled, prospective trials, and issues with data generalizability.

Summary Antibiotic overuse may improve when mPCR is coupled with electronic medical record algorithm-based approaches and direct provider feedback by an antimicrobial stewardship professional. mPCR may prove a useful tool for antimicrobial stewardship but future studies are needed to define the best practice for its utilization.
\end{abstract}

Keywords Multiplex PCR · Syndromic panel · Antimicrobial stewardship · Molecular diagnostics

\section{Introduction}

Since the approval by the FDA of the first multiplex Polymerase Chain Reaction (mPCR) panel for the detection

This article is part of the Topical Collection on Healthcare Associated Infections

Theodore S. Rader, IV

theodore.rader@vcuhealth.org

Michael P. Stevens

Michael.stevens@vcuhealth.org

Gonzalo Bearman

Gonzalo.Bearman@vcuhealth.org

1 Department of Internal Medicine, Virginia Commonwealth University Health System, 1250 E MARSHALL ST \# 980509, Richmond, VA 23298-0019, USA

2 Division of Infectious Diseases, Virginia Commonwealth University Health System, Richmond, VA, USA of respiratory pathogens, the BioFire Respiratory Pathogen Panel, the landscape of syndromic multiplex PCR panel testing continues to evolve $[1,2]$. These tests, which subsequently expanded to include gastrointestinal (GI), bloodstream, and central nervous system (CNS) syndromic panels in addition to the respiratory panel, promise rapid turnaround times with high sensitivity and specificity when compared to more traditional methods such as culture [3]. Clinicians can gain rapid information regarding the nature of a patient's illness to guide clinical care, such as the timely initiation of targeted antimicrobials. Challenges remain in defining the best utilization of these syndromic panels in clinical care, and while many practice guidelines contain statements advising consideration of PCR-based diagnosis as part of the diagnostic workup, there are no recommendations provided on the best utilization of these panels [4, 5].

Additionally, global concern about the rise of antimicrobial resistance driven by antibiotic overprescribing continues to increase. Syndromic mPCR panels offer rapid information useful to target or remove antimicrobials in the clinical context 
of a patient. The Society for Healthcare Epidemiology of America (SHEA) recently released a white paper outlining research needs for antimicrobial stewardship. Among the many suggested high-value targets for stewardship, mPCR has obvious benefits to drive practice changes in the prescription of antibiotics via diagnostic stewardship. This review provides an overview of the antimicrobial stewardship intervention landscape for respiratory, GI, and CNS panels. It will examine the types of studies conducted, the methods taken for stewardship, and the results of those efforts in improving the appropriate use of antibiotics.

\section{Respiratory Multiplex Pathogen Panels and Antimicrobial Stewardship}

The introduction of the BioFire FilmArray Respiratory Pathogen panel in 2011 marked a new era of rapid diagnostic testing for respiratory viral and bacterial pathogens. Since then, several other panels have been approved, including the Verigene, Luminex x-TAG series, eSensor Respiratory Virus Panel (RVP), and ePlex systems. This review is not meant to be a comprehensive review of the technical details of respiratory mPCR panels, and more comprehensive technical reviews are available elsewhere $[1,6]$. In general, the sensitivities and specificities of the panels vary by which test is used and what virus is tested. The BioFire Respiratory Pathogen panel has nearly 99-100\% specificity for the viruses included, but the sensitivity for certain viruses ranges from 57-100\% depending on the study examined and the virus in question. In one example, Popwich et al. found that the FilmArray Respiratory Pathogen panel was $57 \%$ sensitive for adenovirus, whereas Rand et al. found sensitivity of $90 \%[7,8]$. For the eSensor RVP, the reported sensitivities range from 92 to $100 \%$ with specificity of $100 \%$ [1, 7]. For the $\mathrm{x}$-TAG series, sensitivity and specificity are reported by panel types $x-T A G$ RVP, $x-T A G$ RVP Fast, and Nx-TAG. NxTAG is the current version of rapid syndromic $\mathrm{MPCR}$ available from Luminex, and reported sensitivity is $93-100 \%$ for most viruses with the exception of coronavirus HKU1 and OC43, with reported sensitivities of $67 \%$ each. Additionally calculated specificity is $>97 \%$ for all included viral pathogens in this study [9]. Examining the underlying data, low sample numbers may limit interpretation of these results for the Nx-TAG, with only 3 positives in the sample set for coronavirus HKU1 and OC43. Generally, mPCR for respiratory pathogens should be considered sensitive and specific for identifying respiratory pathogens.

One advantage syndromic mPCR panels offer over more traditional methods is in the turnaround time of the panel. Depending on the collection method and timing for sample processing, the turnaround time can be as low as $1.5 \mathrm{~h}$, but can be longer if samples are run in a batched fashion [10-17]. This faster turnaround time translates into better antibiotic prescribing in some cases; in one example, a multiplex influenza/RSV assay changed the antimicrobials prescribed in $58 \%$ of patients in an emergency room setting, decreasing inappropriate antibiotic and antiviral prescriptions by $24.5 \%$ and $9 \%$, respectively [12]. In the context of identifying influenza infections, multiple studies showed influenza virus-positive patients receive fewer antibiotics than influenza-negative patients [11-13]. More of these patients also receive oseltamivir or other antiviral medications, and $\mathrm{mPCR}$ reduces the time to delivery of antiviral medications [17]. The absence of influenza or the positive presence of another pathogen did not significantly change the rate of antibiotics prescribed, and there was no difference in the rates of antibiotics, $48.6 \%$ vs $49.3 \%$ in one study. Of the patients with a positive viral panel for influenza or other non-influenza viruses, $32 \%$ still received antibiotics [17]. In the ResPOC trial, one of the few randomized controlled trials pertaining to stewardship and $\mathrm{mPCR}$ panels, patients were randomized over two respiratory virus seasons to utilize the BioFire Respiratory Panel or an in-house PCR to determine the effect on antibiotic utilization. No significant differences were found in the number of patients who received antibiotics or the mean duration of antibiotics. Importantly, significantly fewer patients in the mPCR group than the control group received more than one dose of antibiotics [18]. Implementation of an MPCR respiratory panel alone appears not to have a replicable significant impact on antibiotic use or duration of antibiotics.

Adding an educational intervention to the stewardship process may reduce antimicrobial use. In a retrospective study by Keske et al., an educational intervention conducted during the implementation of a respiratory $\mathrm{mPCR}$ panel significantly increased the rate of discontinued antibiotics and overall decreased total antibiotic prescriptions [19]. In a randomized controlled trial by Branche et al., investigators educated providers on how to utilize an mPCR panel and procalcitonin (PCT) level algorithm for antibiotic de-escalation prior to the implementation of the study and were sent reminders of the algorithm on patient enrollment. While no difference was found in the duration of antibiotics overall, subsequent analysis found that algorithm adherent patients received significantly fewer days of antibiotics and fewer algorithm patients received antibiotics at discharge. The authors note that providers prescribed antibiotics for patients with high procalcitonin values more often than they withheld antibiotics for patients with low procalcitonin values [20]. In subsequent analysis, an admitting diagnosis of pneumonia was associated with overruling the algorithm in patients, with $7 \%$ of patients in the algorithm-adherent category carrying this diagnosis vs. $26 \%$ of patients in the nonadherent group [21]. Viewing these results, there may be ways to educate and promote adherence to directed therapy, such as encouraging more direct algorithm adherence, or providing education on viral pneumonias. 
Adding a directed intervention by an external reviewer can improve antimicrobial stewardship using mPCR. In a prospective study by Lowe et al., an antimicrobial stewardship program (ASP) provider reviewed the patient's chart and the results of an mPCR panel to determine if a patient would benefit from an ASP consult to provide recommendations in an audit-and-feedback model. They achieved successful reduction of 1.3 days of antibiotics in the prospective ASP audit cohort, and if their recommendations were accepted, 3.6 fewer days of antibiotics [22]. Similarly, Abbas et al. at Virginia Commonwealth University Health System reported success with an ASP provider intensive measure. An ASP member contacted inpatient provider teams about patients with positive results from a respiratory MPCR panel and made recommendations to de-escalate, change, or discontinue antibiotics. The primary teams followed the recommendations of the ASP provider $66 \%$ of the time overall; however, when the recommendation was discontinuation or de-escalation, providers accepted $19 \%$ of the time [23]. More recently, the Cleveland Clinic Foundation Health system undertook a large, multisite trial of an ASP provider recommendation for patients with a positive mPCR panel. Of their interventions, primary teams followed only $47 \%(n=26)$ of the 55 recommendations. This did not translate into a significantly reduced duration of antibiotic therapy, and intervention of an ASP provider did not influence the de-escalation of any one antibiotic $[24 \bullet \bullet]$. These provider intensive interventions require the time of a trained professional to review charts and make recommendations to the primary medicine team, but a large percentage of recommendations are not accepted, which may be a limitation of this method and may not represent an appropriate utilization of stewardship resources.

In a possible demonstration of a rationale to overcome a stewardship provider intensive method, Moradi et al. combined several methods to reduce antibiotic use without the intervention of a trained provider. Patients in a pre-post quasi-experimental study with a PCT $\leq 0.25 \mathrm{ng} / \mathrm{ml}$, a mPCR panel positive for 1 virus, and at least 1 systemic antibiotic automatically triggered an alert in the Electronic Medical Record (EMR) to display when any provider opened the chart. This "Best Practices Alert" (BPA) displayed for the provider the PCT value, mPCR results, and the active antibiotics. The provider was given options to suppress the alert temporarily or permanently, and the interactions by providers with this alert were tracked. Patients in the intervention group who triggered the alert had their average days of antibiotics reduced significantly by 2.2 days $(5.8$ vs 8.0$)$. In the intervention group, more antibiotics were discontinued within $24 \mathrm{~h}$ of initiation, there were fewer days of antibiotic therapy, and fewer antibiotic prescriptions on discharge [25••]. Within the different strategies to improve antibiotic discontinuation, it appears that utilization of an EMR base practice alert, possibly in conjunction with procalcitonin, can improve the use of respiratory mPCR panels for stewardship.

\section{Gastrointestinal Multiplex Pathogen Panels and Antimicrobial Stewardship}

When compared to respiratory pathogen $\mathrm{MPCR}$, fewer gastrointestinal (GI) mPCR stewardship investigations exist. Available panels include the BioFire FilmArray Gastrointestinal Panel, Luminex xTAG Gastrointestinal Pathogen Panel, and Verigene enteric pathogens panel. Like other mPCR panels, GI mPCR provides a faster turnaround time when compared to traditional methods such as stool culture and examination for ova and parasites [26, 27•, 28]. mPCR panels detect pathogens $10-36 \%$ more often than traditional methods depending on the panel used. Sensitivity for the BioFire FilmArray GI mPCR ranged from $94.5 \%$ for 7 of the 22 pathogens in the panel to $100 \%$ for 10 of 22 pathogens in one study. Specificity ranged from $93.4-100 \%$ depending on the target organism in the same study [29]. In the only direct comparison of the three enteric pathogen MPCR panels, the Verigene enteric pathogen $\mathrm{mPCR}$ panel reported sensitivity and specificity for Campylobacter of $83.3 \%$ and $99.3 \%$, Salmonella $83.3 \%$ and $100 \%$, Shigella $95.4 \%$ and $99.1 \%$, Shiga-Toxin producing Escherichia coli $91.7 \%$ and $100 \%$, norovirus $89.0 \%$ and $100 \%$, and rotavirus of $71.4 \%$ and $100 \%$. The Luminex panel analyzed in this study reported sensitivities of $91.7 \%$ for Campylobacter, $79.2 \%$ for Salmonella, $100 \%$ for Shigella, $91.7 \%$ for Shiga-Toxin producing Escherichia coli, $89.5 \%$ for norovirus, and $100 \%$ for rotavirus, with $100 \%$ specificity for all targets. The BioFire FilmArray mPCR was $94.7-100 \%$ sensitive for these targets, and $98.6-100 \%$ specific [30].

In a large retrospective analysis, patients whose diagnostic workup included mPCR received fewer antibiotics $(36.2 \%$ vs 40.9\%). Patients with PCR testing were also less likely to undergo additional endoscopy or imaging. There were important differences between the two groups in this study. The mPCR group was larger, older and more likely to be inpatient, limiting the interpretation of the true effect size of the mPCR intervention [31].

Similar to respiratory mPCR panels, using a stewardship intervention to educate clinicians on MPCR and appropriate antibiotic use appears to change prescribing habits. This approach helped Keske et al. reduce the inappropriate use of antibiotics from $42.9 \%$ to $25.8 \%$ in the post intervention period. This effect was maintained with a larger sample size in the post-intervention group while still allowing unrestricted access to the testing [32]. In the only prospective trial of GI $\mathrm{mPCR}$, parallel cultures conducted in conjunction with the BioFire GI panel compared antimicrobial use in new inpatient and outpatients, both adults and children, to a historical 
control group. The mPCR panel replaced conventional culture as the orderable test immediately prior to the study period. As the turnaround time of test results decreased, there was a significant trend towards targeted antibiotics being prescribed, and proportionally fewer patients had initiation of antimicrobial therapy empirically. There also appeared to be an increasing use of the test results to target antimicrobial therapy to the organism detected, as over a period of months from the implementation of the test, the increasing utilization of narrowly directed therapy was apparent [27•].

These studies suggest a role in the use of mPCR GI panels for stewardship of antimicrobials and resources, although diagnostic test stewardship is an important consideration. In a retrospective cohort of patients in a single hospital system, patients with an initial negative GI mPCR result were retested within 4 weeks of the initial negative result, and the vast majority remained negative, with no significant rate of conversion to positives [33]. In a subsequent follow up study from the same group, only $3.9 \%$ of tests for patients admitted $>72 \mathrm{~h}$ were positive, ignoring viral shedding and suspected false positives [34]. Taken together, these results suggest that it is reasonable to test patients suspected to have infectious diarrhea with mPCR at least once; however, patients hospitalized for $>72 \mathrm{~h}$ likely do not benefit from testing, and that repeat testing for patients with continued symptoms is unlikely to yield a different result.

\section{Central Nervous System Multiplex Panels}

Currently, the BioFire FilmArray Meningitis/Encephalitis (ME) panel is the only mPCR test approved by the U.S. Food and Drug Administration for cerebrospinal fluid analysis. It evaluates for 14 bacterial, viral, and fungal pathogens and can provide results within $1 \mathrm{~h}$ (https://www.biofiredx. com/products/the-filmarray-panels/filmarrayme/), which has been born out in clinical scenarios with several studies reporting a turnaround time of less than $2 \mathrm{~h}$ [35-37]. This provides inherent advantages over cultures, which can take days to result and can be insensitive while specific [38]. In addition, a recent systematic review and meta-analysis determined the overall sensitivity and specificity to be $90 \%$ (95\% CI 86-93\%) and 97\% (95\% CI 94-99\%) [39]. Advantages in sensitivity, specificity, and turnaround time should allow for quick de-escalation of antimicrobials if results of the PCR are negative, something not noted in several retrospective preand-post implementation studies.

Dack et al. and Chang et al. both conducted retrospective observational studies of the effect of implementation of the BioFire Meningitis-Encephalitis Panel on antimicrobial usage. Both found negative MPCR panel patients had a median duration of 3 and 4 days of antimicrobial therapy, respectively, but did not prove a statistically significant decrease in antimicrobial use $[36,40]$. The reasons given for possible continuation of antimicrobials include clinicians' concerns with mortality, or unfamiliarity with new technologies. In another pre-and-post implementation observational study, Radmard and colleagues learned that despite a rapid turn-around time of $1.5 \mathrm{~h}$, the median time to narrowing or discontinuation of antimicrobial therapy was $26.5 \mathrm{~h}$, with $25 \%$ of patients remaining on empiric therapy 24-48 h after negative panel results. Radmard et al. did not compare these results to the pre-mPCR time period and noted that because of the many factors associated with antimicrobial usage, they could not evaluate the mPCR ME panel for antimicrobial stewardship [37]. Mina et al. did find a significant difference in the duration antimicrobial usage, $9.5 \pm 3.7$ days in the mPCR group and $15.2 \pm 5$ days in the control group $(p=0.007)$. It is important to note the authors only conducted significance assessment on the presence of bacterial meningitis on the MPCR panel versus a control group that could have had non-bacterial meningitis, but did not have a mPCR panel collected [41].

A recent prospective observational trial in France studied the effect of mPCR diagnosis of meningitis/encephalitis on the management of patients with suspected CNS infections. The use of an MPCR panel led to an earlier discontinuation of empiric antimicrobials in $32 \%$ of the cases. The authors additionally observed a reduction in the length of stay for $18 \%$ of patients. A significant limitation to this study is the absence of reporting tests of statistical significance [42•]. Overall, a lack of more generalizable data limits a more nuanced review of CNS mPCR use in antimicrobial stewardship.

Although there is limited evidence that the CNS mPCR panel reduces antimicrobial use, several groups reported potential cost savings that can be achieved by using $\mathrm{MPCR}$ in the diagnosis of CNS infections. DiDodato et al. estimated cost savings based on a standardized cost of stay in Canadian hospitals for CNS infections. Using observational data from preand-post introduction of the $\mathrm{MPCR}$ ME panel at a communitybased university affiliated hospital, they estimated a cost savings per patient case of \$2319 CDN (\$1693 USD), calculated by determining the change in time to discharge. The cost savings in their model were driven by time to definitive microbiologic reporting, which happened earlier in the group utilizing mPCR. Costs of microbiologic diagnosis during the same period were stable and thought to be offset by changes in the standard of care when utilizing mPCR, so the cost of the mPCR panel was not included in the final analysis [43]. In a separate study from France, the reduction in hospital stay from using an mPCR ME panel resulted in estimated savings of 201 $€ /$ patient ( $\sim 245$ USD). Soucek et al. identified cost savings of \$38.73 USD in antimicrobials when using a CNS mPCR panel. When including the cost of diagnostic testing, that gap narrowed to $\angle \$ 1$ USD $(P=0.15)$ [44]. These studies are useful in justifying the cost of the $\mathrm{mPCR}$ panel, which can run $>\$ 100$ USD. 
Table 1 Multiplex PCR and antimicrobial and diagnostic stewardship: key findings

\begin{tabular}{|c|c|c|}
\hline PCR panel pathogen type & Key references & Key findings and author comments \\
\hline Respiratory mPCR panel & $\begin{array}{l}\text { Branche et al. [20], Branche et al. } \\
\text { [21], Keske et al. [19], Abbas et al. [23], } \\
\text { Lowe et al. [26], Srinivas et al. [24••], } \\
\text { Moradi et al. [25••] }\end{array}$ & $\begin{array}{l}\text { - Respiratory mPCR increases more timely antiviral } \\
\text { prescriptions for influenza } \\
\text { - Combining a respiratory mPCR panel with a } \\
\text { procalcitonin-based algorithm can improve stewardship, } \\
\text { but algorithm adherence is critical } \\
\text { - Educational interventions when implementing mPCR } \\
\text { panels may be useful in improving stewardship } \\
\text { - mPCR results are most useful when tied to direct } \\
\text { antimicrobial atewardship activities } \\
\text { - When algorithms incorporating mPCR results are utilized, } \\
\text { assessing end-user guideline adherence is critical for } \\
\text { optimizing and refining these protocols } \\
\text { - Decision support within Electronic Medical Records that } \\
\text { incorporates mPCR results can reduce antibiotic use }\end{array}$ \\
\hline Gastrointestinal mPCR Panel & $\begin{array}{l}\text { Keske et al. [32], Axeldrad et al. [31], } \\
\text { Beal et al. [26], Cybulski et al. [27•] }\end{array}$ & $\begin{array}{l}\text { - GI mPCR are associated with improved test turn-around time } \\
\text { when compared with traditional methods } \\
\text { - GI mPCR can decrease antimicrobial duration } \\
\text { - Educational interventions can optimize the use of GI } \\
\text { mPCR panels } \\
\text { - Diagnostic stewardship is critical as positive results do not } \\
\text { necessarily indicate active infection } \\
\text { - The optimal role of Antimicrobial Stewardship Programs in } \\
\text { PCR test review and intervention needs to be defined }\end{array}$ \\
\hline Central nervous system mPCR panel & $\begin{array}{l}\text { Dack et al. [40], Chang et al. [36], } \\
\text { Radmard et al. [37], Mina et al. [41] }\end{array}$ & $\begin{array}{l}\text { - One mPCR panel, the BioFire FilmArray meningitis-encephalitis } \\
\text { panel, is U.S. FDA approved } \\
\text { - CNS mPCR is associated with improved turnaround time } \\
\text { (over traditional methods) } \\
\text { - mPCR may have suboptimal sensitivity for Cryptococcus } \\
\text { compared to traditional diagnostic testing; this has important } \\
\text { implications for testing and clinical management } \\
\text { - Positive test results for specific viruses have to be interpreted } \\
\text { in context (as these may represent systemic reactivation and } \\
\text { not active infection) } \\
\text { - The optimal role of Antimicrobial Stewardship Programs in } \\
\text { mPCR test review and intervention needs to be defined } \\
\text { - CNS mPCR is promising but more research is needed }\end{array}$ \\
\hline
\end{tabular}

Additionally, several issues relating to the interpretation of CNS mPCR results have not been resolved. Several papers highlight concerns that the sensitivity of the BioFire CNS mPCR for Cryptococcus neoformans/gattii may be suboptimal when compared to traditional methods [37, 45-48]. However, a recent systematic review of cases reported in the literature found that most reported cryptococcus results lacked an appropriate comparator method or were compared to only a serum cryptococcal antigen, concluding that there was a higher negative predictive value to the MPCR panel than individual studies suggested, with an overall sensitivity and specificity of $>90 \%$ [39]. Finally, positive Human Herpes Virus 6 (HHV-6), Cytomegalovirus (CMV), and Herpes Simplex Virus (HSV) results must be interpreted in the appropriate context, as reactivation and active infection can both result as positive $[39,47]$. At this time, mPCR CNS panels are promising for stewardship, but more study is needed to define best practices for their use.

\section{Future Directions for Multiplex PCR Panels in Antimicrobial Stewardship}

The use of mPCR panels to guide antimicrobial therapy and enhance antimicrobial stewardship shows promise, but more studies are needed to define their most optimal use. Table 1 summarizes the key findings from our review of stewardship literature related to respiratory, GI and CNS mPCR panels. If mPCR panels are adopted, incorporation of these technologies in local treatment guidelines is critical. Guidelines help frontline clinicians best utilize these tests and help reinforce best antimicrobial management practices. These algorithms may or may not include procalcitonin, as recently, the diagnostic sensitivity and specificity of elevated procalcitonin for distinguishing viral from bacterial pneumonia has been called into question [49].

Another way to optimize the use of MPCR is via EMRbased decision support. Moradi et al. did this via adding a Best 
Practice Alert into their EMR [25••]. EMR tools can activate based on pre-set data criteria and can provide guidance directly to providers. Adding a EMR stewardship visualization tool worked for a Veterans Affairs Health System Hospital and could be combined with any one of the mPCR panels to improve utilization of antibiotics [50].

ASP personnel can actively review new mPCR results and provide best practice recommendations to providers directly. These efforts are most useful when ASP personnel are acting on these data in real-time $[22,23,24 \cdot \bullet]$. Compliance with ASP recommendations should be monitored and feedback to end users provided. When compliance is suboptimal, the reasons for this should be explored. ASP interventions utilizing realtime mPCR data are labor intensive and have significant implications for program staffing and resources.

In assessing the effectiveness of treatment guidelines incorporating $\mathrm{mPCR}$ panels, surveying providers about their rationale for following an algorithm (or not) may be useful to help optimize the use of these technologies. Importantly, providing feedback to providers about rates of antibiotic prescribing may improve subsequent antibiotic use [51].

For GI and CNS mPCR panels, there are no published, peer-reviewed studies available at the time of this publication that explore the use of EMR-based decision support in the context of antimicrobial stewardship. Extrapolating from respiratory mPCR studies, EMR-based decision support may optimize the use of these technologies. For both GI and respiratory mPCR panels, diagnostic test stewardship (via test restriction) appears critically important $[34,52,53]$.

\section{Conclusions}

Respiratory, GI and CNS syndromic mPCR panels, as an isolated intervention, aid in the diagnosis of infectious pathogens yet do not consistently improve antimicrobial utilization. Wherever possible, these technologies should be incorporated into local treatment guidelines with active antimicrobial stewardship program oversight and support. The use of EMRbased decision support is also promising. Provider compliance with guidelines utilizing mPCR panels should be monitored and feedback should be provided. Additional research is needed to define best practices for the use of MPCR panels in both antimicrobial and diagnostic test stewardship.

\section{Declarations}

Human and Animal Rights and Informed Consent All reported studies/ experiments with human or animal subjects performed by the authors have been previously published and complied with all applicable ethical standards (including the Helsinki declaration and its amendments, institutional/national research committee standards, and international/national/institutional guidelines).
Conflict of interest Theodore S. Rader, Michael P. Stevens, and Gonzalo Bearman declare that they have no conflict of interest.

\section{References}

Papers of particular interest, published recently, have been highlighted as:

- Of importance

•- Of major importance

1. Ramanan P, Bryson AL, Binnicker MJ, Pritt BS, Patel R. Syndromic panel-based testing in clinical microbiology. Clin Microbiol Rev [Internet]. American Society for Microbiology Journals; 2018 [cited 2020 Mar 25];31. Available from: https:// cmr.asm.org/content/31/1/e00024-17.

2. BioFire Receives FDA Clearance for FilmArray ${ }^{\circledR}$ Torch with FilmArray ${ }^{\circledR}$ Respiratory Panel and Submits Special 510(k) Applications for use with additional FilmArray ${ }^{\circledR}$ Panels [Internet]. BioMérieux Corp. Website. [cited 2016 Dec 10]. Available from: http://www.biomerieux.com/en/biofire-receivesfda-clearance-filmarrayr-torch-filmarrayr-respiratory-panel-andsubmits-special.

3. Couturier MR, Bard JD. Direct-from-specimen pathogen identification: evolution of syndromic panels. Clin Lab Med. 2019;39: 433-51.

4. Metlay JP, Waterer GW, Long AC, Anzueto A, Brozek J, Crothers $\mathrm{K}$, et al. Diagnosis and treatment of adults with communityacquired pneumonia. An Official Clinical Practice Guideline of the American Thoracic Society and Infectious Diseases Society of America. Am J Respir Crit Care Med. 2019;200:e45-67.

5. Shane AL, Mody RK, Crump JA, Tarr PI, Steiner TS, Kotloff K, et al. 2017 Infectious Diseases Society of America Clinical Practice Guidelines for the Diagnosis and Management of Infectious Diarrhea. Clin Infect Dis. 2017;65:e45-80.

6. Poritz MA, Lingenfelter B. Multiplex PCR for Detection and identification of microbial pathogens. In: Tang Y-W, Stratton CW, editors. Adv Tech Diagn Microbiol Vol 2 Appl [Internet]. Cham: Springer International Publishing; 2018 [cited 2020 Mar 27]. 2018. p. 475-93. Available from: https://doi.org/10.1007/978-3319-95111-9 19.

7. Popowitch EB, O'Neill SS, Miller MB. Comparison of the Biofire FilmArray RP, Genmark eSensor RVP, Luminex xTAG RVPv1, and Luminex xTAG RVP Fast Multiplex Assays for Detection of Respiratory Viruses. J Clin Microbiol. 2013;51:1528-33.

8. Rand KH, Rampersaud H, Houck HJ. Comparison of two multiplex methods for detection of respiratory viruses: FilmArray RP and xTAG RVP $\nabla$. J Clin Microbiol. 2011;49:2449-53.

9. Chen JHK, Lam H-Y, Yip CCY, Wong SCY, Chan JFW, Ma ESK, et al. Clinical evaluation of the new high-throughput Luminex NxTAG Respiratory Pathogen Panel assay for multiplex respiratory pathogen detection. J Clin Microbiol. 2016:JCM.00517-16.

10. Xu M, Qin X, Astion ML, Rutledge JC, Simpson J, Jerome KR, et al. Implementation of FilmArray respiratory viral panel in a core laboratory improves testing turnaround time and patient care. Am J Clin Pathol. 2013;139:118-23.

11. Rappo U, Schuetz AN, Jenkins SG, Calfee DP, Walsh TJ, Wells MT, et al. Impact of early detection of respiratory viruses by multiplex PCR assay on clinical outcomes in adult patients. J Clin Microbiol. 2016;54:2096-103.

12. Hansen GT, Moore J, Herding E, Gooch T, Hirigoyen D, Hanson $\mathrm{K}$, et al. Clinical decision making in the emergency department 
setting using rapid PCR: results of the CLADE study group. J Clin Virol. 2018;102:42-9.

13. Green DA, Hitoaliaj L, Kotansky B, Campbell SM, Peaper DR. Clinical utility of on-demand multiplex respiratory pathogen testing among adult outpatients. J Clin Microbiol. 2016;54:2950-5.

14. Gelfer G, Leggett J, Myers J, Wang L, Gilbert DN. The clinical impact of the detection of potential etiologic pathogens of community-acquired pneumonia. Diagn Microbiol Infect Dis. 2015;83:400-6.

15. Gardiner BJ, Parker CEV, Rabson AR, Snydman DR, Doron S. Evaluation of a two-stage testing algorithm for the diagnosis of respiratory viral infections. Diagn Microbiol Infect Dis. 2018;91: 319-23.

16. Echavarría M, Marcone DN, Querci M, Seoane A, Ypas M, Videla $\mathrm{C}$, et al. Clinical impact of rapid molecular detection of respiratory pathogens in patients with acute respiratory infection. J Clin Virol. 2018;108:90-5.

17. Andrews D, Chetty Y, Cooper BS, Virk M, Glass SK, Letters A, et al. Multiplex PCR point of care testing versus routine, laboratorybased testing in the treatment of adults with respiratory tract infections: a quasi-randomised study assessing impact on length of stay and antimicrobial use. BMC Infect Dis. 2017;17:671.

18. Brendish NJ, Malachira AK, Armstrong L, Houghton R, Aitken S, Nyimbili E, et al. Routine molecular point-of-care testing for respiratory viruses in adults presenting to hospital with acute respiratory illness (ResPOC): a pragmatic, open-label, randomised controlled trial. Lancet Respir Med. 2017;5:401-11.

19. Keske S,, Ergönül Ö, Tutucu F, Karaaslan D, Palaoğlu E, Can F. The rapid diagnosis of viral respiratory tract infections and its impact on antimicrobial stewardship programs. Eur J Clin Microbiol Infect Dis. 2018:37:779-83.

20. Branche AR, Walsh EE, Vargas R, Hulbert B, Formica MA, Baran $A$, et al. Serum procalcitonin measurement and viral testing to guide antibiotic use for respiratory infections in hospitalized adults: a randomized controlled trial. J Infect Dis. 2015;212:1692-700.

21. Branche AR, Walsh EE, Jadhav N, Karmally R, Baran A, Peterson $\mathrm{DR}$, et al. Provider decisions to treat respiratory illnesses with antibiotics: insights from a randomized controlled trial. PLoS ONE [Internet]. 2016 [cited 2016 Oct 26];11. Available from: http:// www.ncbi.nlm.nih.gov/pmc/articles/PMC4820114/.

22. Lowe CF, Payne M, Puddicombe D, Mah A, Wong D, Kirkwood A, et al. Antimicrobial stewardship for hospitalized patients with viral respiratory tract infections. Am J Infect Control. 2017;45:8725.

23. Abbas S, Bernard S, Lee KB, Pakyz A, Doern C, Doll M, et al. Rapid respiratory panel testing: impact of active antimicrobial stewardship. Am J Infect Control. 2019;47:224-5.

24.• Srinivas P, Rivard KR, Pallotta AM, Athans V, Martinez K, Loutzenheiser $\mathrm{S}$, et al. Implementation of a stewardship initiative on respiratory viral PCR-based antibiotic deescalation. Pharmacother J Hum Pharmacol Drug Ther. 2019;39:709-17 Important as a directed stewardship intervention across a large hospital system looking at positive Respiratory MPCR and providing direct feedback to the ordering teams via trained antimicrobial stewardship provider.

25.• Moradi T, Bennett N, Shemanski S, Kennedy K, Schlachter A, Boyd S. Use of procalcitonin and a respiratory polymerase chain reaction panel to reduce antibiotic use via an EMR alert. Clin Infect Dis [Internet]. 2019 [cited 2019 Oct 29]; Available from: https:// academic.oup.com/cid/advance-article/doi/10.1093/cid/ciz1042/ 5601956. Recent quasi-experimental pre-and-post implementation study utilizing a best-practice alert to provide ordering clinicians with evidenced-based guidance on procalcitoninguided antimicrobial stewardship. Clinicians were alerted to the guidance when certain conditions were met in the electronic medical record. Decreased antimicrobials in a prospective manner through a targeted intervention.

26. Beal SG, Tremblay EE, Toffel S, Velez L, Rand KH. A Gastrointestinal PCR panel improves clinical management and lowers health care costs. J Clin Microbiol. 2018;56.

27. Cybulski RJ, Bateman AC, Bourassa L, Bryan A, Beail B, Matsumoto J, et al. Clinical impact of a multiplex gastrointestinal polymerase chain reaction panel in patients with acute gastroenteritis. Clin Infect Dis. 2018;67:1688-96 Prospective multicenter study with large sample size compared to historic control that examined the impact of the BioFire GI panel on clinical resource utilization. Turnaround time and pathogen detection as percent of samples tested both improved with GI PCR. Fewer patients in the mPCR group had empiric antibiotics and there was a significant trend towards targeted over empiric therapy. Demonstrates potential of GI mPCR for antimicrobial stewardship in clinical practice.

28. Robilotti E, Powell E, Usiak S, Taur Y, Babady NE, Kamboj M. The Perils of Multiplex Gastrointestinal pathogen panels: pseudooutbreaks of Salmonellae and Entamoeba histolytica in Immunocompromised Hosts. Infect Control Hosp Epidemiol. 2018;39:867-70.

29. Buss SN, Leber A, Chapin K, Fey PD, Bankowski MJ, Jones MK, et al. Multicenter evaluation of the BioFire FilmArray gastrointestinal panel for etiologic diagnosis of infectious gastroenteritis. J Clin Microbiol. 2015;53:915-25.

30. Huang RSP, Johnson CL, Pritchard L, Hepler R, Ton TT, Dunn JJ. Performance of the Verigene $\AA$ enteric pathogens test, Biofire FilmArray ${ }^{\mathrm{TM}}$ gastrointestinal panel and Luminex xTAG ${ }^{\circledR}$ gastrointestinal pathogen panel for detection of common enteric pathogens. Diagn Microbiol Infect Dis. 2016;86:336-9.

31. Axelrad JE, Freedberg DE, Whittier S, Greendyke W, Lebwohl B, Green DA. Impact of gastrointestinal panel implementation on health care utilization and outcomes. J Clin Microbiol. 2019;57: e01775-18.

32. Keske Ş, Zabun B, Aksoy K, Can F, Palaoğlu E, Ergönül Ö. Rapid molecular detection of gastrointestinal pathogens and its role in antimicrobial stewardship. J Clin Microbiol [Internet]. 2018 [cited 2019 Oct 13];56. Available from: https://www.ncbi.nlm.nih.gov/ pmc/articles/PMC5925700/.

33. Park S, Hitchcock MM, Gomez CA, Banaei N. Is follow-up testing with the FilmArray gastrointestinal multiplex PCR panel necessary? J Clin Microbiol. 2017;55:1154-61.

34. Hitchcock MM, Gomez CA, Banaei N. Low yield of FilmArray GI panel in hospitalized patients with diarrhea: an opportunity for diagnostic stewardship intervention. J Clin Microbiol [Internet]. 2018 [cited 2019 Oct 13];56. Available from: https://www.ncbi.nlm.nih. gov/pmc/articles/PMC5824048/.

35. Boudet A, Pantel A, Carles M-J, Boclé H, Charachon S, Enault C, et al. A review of a 13-month period of FilmArray Meningitis/ Encephalitis panel implementation as a first-line diagnosis tool at a university hospital. PLoS ONE. 2019;14:e223887.

36. Chang D, Okulicz JF, Nielsen LE, White BK. A tertiary care center's experience with novel molecular meningitis/encephalitis diagnostics and implementation with antimicrobial stewardship. Mil Med. 2018;183:e24-7.

37. Radmard S, Reid S, Ciryam P, Boubour A, Ho N, Zucker J, et al. Clinical utilization of the FilmArray meningitis/encephalitis (ME) multiplex polymerase chain reaction (PCR) assay. Front Neurol [Internet]. 2019 [cited 2019 Oct 12];10. Available from: https:// www.ncbi.nlm.nih.gov/pmc/articles/PMC6443843/.

38. Brouwer MC, Tunkel AR, van de Beek D. Epidemiology, diagnosis, and antimicrobial treatment of acute bacterial meningitis. Clin Microbiol Rev. 2010;23:467-92. 
39. Tansarli GS, Chapin KC. Diagnostic test accuracy of the BioFire ${ }^{\circledR}$ FilmArray ${ }^{\circledR}$ meningitis/encephalitis panel: a systematic review and meta-analysis. Clin Microbiol Infect. 2020;26:281-90.

40. Dack K, Pankow S, Ablah E, Zackula R, Assi M. Contribution of the BioFire ${ }^{\circledR}$ FilmArray ${ }^{\circledR}$ meningitis/encephalitis panel: assessing antimicrobial duration and length of stay. Kans J Med. 2019;12:13 .

41. Mina Y, Schechner V, Savion M, Yahav D, Bilavsky E, Sorek N, et al. Clinical benefits of FilmArray meningitis-encephalitis PCR assay in partially-treated bacterial meningitis in Israel. BMC Infect Dis [Internet]. 2019 [cited 2019 Oct 28];19. Available from: https:// www.ncbi.nlm.nih.gov/pmc/articles/PMC6693273/.

42. Cailleaux M, Pilmis B, Mizrahi A, Lourtet-Hascoet J, Van JCN, Alix L, et al. Impact of a multiplex PCR assay (FilmArray®) on the management of patients with suspected central nervous system infections. Eur J Clin Microbiol Infect Dis. 2020;39:293-7 Examined prospectively the impact of ME MPCR on factors associated with positivity, time to discharge, and provided quantitative data on discontinuation of empiric antibiotics.

43. DiDiodato G, Bradbury N. Cerebrospinal fluid analysis with the BioFire FilmArray meningitis/encephalitis molecular panel reduces length of hospital stay in patients with suspected central nervous system infections. Open Forum Infect Dis [Internet]. 2019 [cited 2019 Oct 28];6. Available from: https://www.ncbi.nlm.nih.gov/ pmc/articles/PMC6483125/.

44. Soucek DK, Dumkow LE, VanLangen KM, Jameson AP. Cost justification of the BioFire FilmArray meningitis/encephalitis panel versus standard of care for diagnosing meningitis in a community hospital. J Pharm Pract. 2019;32:36-40.

45. Chew KL, Lee CK, Cross GB, Lum LHW, Yan B, Jureen R. Culture-confirmed cryptococcal meningitis not detected by Cryptococcus PCR on the Biofire meningitis/encephalitis panel®. Clin Microbiol Infect. 2018;24:791-2.

46. Liesman RM, Strasburg AP, Heitman AK, Theel ES, Patel R, Binnicker MJ. Evaluation of a commercial multiplex molecular panel for diagnosis of infectious meningitis and encephalitis. $\mathrm{J}$ Clin Microbiol. 2018;56:e1927-17.
47. Leber AL, Everhart K, Balada-Llasat J-M, Cullison J, Daly J, Holt $\mathrm{S}$, et al. Multicenter evaluation of BioFire FilmArray meningitis/ encephalitis panel for detection of bacteria, viruses, and yeast in cerebrospinal fluid specimens. J Clin Microbiol. 2016;54:2251-61.

48. Lewis PO, Lanier CG, Patel PD, Krolikowski WD, Krolikowski MA. False negative diagnostic errors with polymerase chain reaction for the detection of cryptococcal meningoencephalitis. Med Mycol [Internet]. [cited 2020 Mar 20]; Available from: http:// academic.oup.com/mmy/advance-article/doi/10.1093/mmy/ myz064/5520397.

49. Kamat IS, Ramachandran V, Eswaran H, Guffey D, Musher DM. Procalcitonin to distinguish viral from bacterial pneumonia: a systematic review and meta-analysis. Clin Infect Dis. 2020;70:538-42.

50. Graber CJ, Jones MM, Goetz MB, Madaras-Kelly K, Zhang Y, Butler JM, et al. Decreases in antimicrobial use associated with multihospital implementation of electronic antimicrobial stewardship tools. Clin Infect Dis [Internet]. 2019 [cited 2019 Nov 1]; Available from: https://academic.oup.com/cid/advance-article/doi/ 10.1093/cid/ciz941/5610861.

51. Persell SD, Doctor JN, Friedberg MW, Meeker D, Friesema E, Cooper A, et al. Behavioral interventions to reduce inappropriate antibiotic prescribing: a randomized pilot trial. BMC Infect Dis. 2016;16:373.

52. Mandelia Y, Procop GW, Richter SS, Worley S, Liu W, Esper F. Optimal timing of repeat multiplex molecular testing for respiratory viruses. J Clin Microbiol [Internet]. 2020 [cited 2020 Feb 20];58. Available from: https://jcm.asm.org/content/58/2/e01203-19.

53. Marcelin JR, Brewer C, Beachy M, Lyden E, Winterboer T, Murphy $\mathrm{CN}$, et al. Hardwiring diagnostic stewardship using electronic ordering restrictions for gastrointestinal pathogen testing. Infect Control Hosp Epidemiol. 2019;40:668-73.

Publisher's NotePublisher's Note Springer Nature remains neutral with regard to jurisdictional claims in published maps and institutional affiliations. 